\title{
Bogotá bilingüe: tensión entre política, currículo y realidad escolar
}

Yamith José Fandiño-Parra

Universidad de La Salle, Colombia

yfandino@unisalle.edu.co

\section{Resumen}

Este artículo hace un acercamiento histórico y conceptual al marco normativo, la politica bilingüe y los lineamientos curriculares del Programa Bogotá Bilingüe (PBB) y revisa la tensión entre política lingüistica, lineamientos curriculares y realidades escolares.

Como resultado se propone la adopción de un modelo sociocultural crítico que permita reconfigurar procesos y prácticas bilingües.

\section{Palabras clave}

Educación bilingüe, políticas educativas, currículo de bilingüismo, enseñanza de segunda lengua, Bogotá-Colombia. (Fuente: Tesauro de la Unesco).

Recepción: 2013-11-08 / Envío a pares: 2014-06-19 / Aceptación por pares: 2014-07-23 / Aprobación: 2014-08-12 DOI: $10.5294 /$ edu.2014.17.2.1

Para citar este artículo / To reference this article / Para citar este artigo

Fandiño-Parra, Y. J. (2014). Bogotá bilingüe: tensión entre política, currículo y realidad escolar. Educ. Educ. 17 (2), 215-236. Doi. 10.5294/ edu.2014.17.2.1 


\title{
Bilingual Bogotá: Tension between Policy, Curriculum and Actual Conditions in the Schools
}

\begin{abstract}
The article takes a historical and conceptual approach to the regulatory framework, bilingual policy and curriculum guidelines of the Bogotá Bilingual Program (PBB) and looks at the tension between linguistic policy, curriculum guidelines and actual conditions in the schools. The adoption of a critical socio-cultural model to reshape bilingual processes and practices is proposed on the basis of that analysis.
\end{abstract}

\section{Key words}

Bilingual education, education policies, curriculum for bilingualism, teaching a second language, Bogotá-Colombia. (Source: Unesco Thesaurus). 


\section{Bogotá bilíngue: tensão entre política, currículo e realidade escolar}

Resumo

Este artigo faz uma aproximação histórica e conceitual ao marco normativo, à politica bilíngue e aos lineamentos curriculares do Programa Bogotá Bilíngue (PBB). Além disso, revisa a tensão entre política linguística, lineamentos curriculares e realidades escolares. Como resultado, propõe-se a adoção de um modelo sociocultural crítico que permita reconfigurar processos e práticas bilingues.

Palavras-chave

Educação bilíngue, politicas educativas, currículo de bilinguismo, ensino de língua estrangeira, Bogotá-Colômbia. (Fonte: Tesauro da Unesco). 


\section{Introducción}

Con el objetivo de mejorar la calidad de la educación colombiana, el Estado ha emprendido una serie de proyectos encaminados a asegurar la coherencia y la articulación de todos los niveles del sistema educativo. En este contexto, el Programa Nacional de Bilingüismo (PNB) ha buscado consolidarse como un proyecto estratégico para el mejoramiento de la calidad educativa; en particular, para el mejoramiento de la enseñanza de las lenguas extranjeras en Colombia. Desde el año 2012 el PNB se denomina Programa de Fortalecimiento al Desarrollo de Competencias en Lenguas Extranjeras (PFDCLE) y sus tres líneas de acción principales giran en torno a formación docente (cursos de inmersión, cursos virtuales de lengua, mejoramiento de metodología), direccionamiento institucional (encuentros regionales, formulación de proyectos, mejoramiento de inglés en Escuelas Normales Superiores) y evaluación (pruebas diagnósticas y caracterización de los profesores de inglés del país)'.

Sin embargo, algunas cuestiones pertinentes al diseño y la implementación del PNB han sido abordadas de manera crítica por varios investigadores colombianos. Cárdenas (2006), por ejemplo, cuestiona la idoneidad de las condiciones del país para el bilingüismo, por cuanto se establecen pocas horas para la enseñanza del inglés, hay escasez de materiales y profesores calificados, las clases son numerosas y, en general, existen pocas oportunidades para usar inglés en comunicación auténtica. Al respecto, Usma (2009) afirma que al igual que otras reformas educativas, el PNB trae consigo oportunidades para algunos grupos e individuos pero genera mayoritariamente procesos de desigualdad, exclusión y estratificación social con los nuevos discursos y prácticas que se adoptan. Para él, la noción instrumentalista del bilingüismo español-inglés no solo excluye las lenguas indígenas y las demás lenguas extranjeras,

$1 \quad$ En este artículo se hablará del PNB en vez del PFDCLE, puesto que este cambio no solo es reciente sino que hasta el momento no se observan cambios sustanciales en la postura conceptual y el enfoque metodológico del Ministerio de Educación Nacional. sino que impone discursos y prácticas importadas al país en detrimento del conocimiento local.

Por su parte, Torres-Martínez (2009) sostiene que la enseñanza-aprendizaje de lenguas extranjeras en Colombia está enmarcada por un conflicto entre lo legal y lo operativo. Este enfrentamiento ha sido resultado de decisiones que se toman con base en estándares internacionales y que se operacionalizan a través de acciones locales sin medios y recursos suficientes para realizar cambios reales. Para él, este conflicto se ha acentuado con una producción de programas, decretos y planes de desarrollo ajustados a los ideales de mejoramiento de los gobiernos nacionales y locales de turno. En consecuencia, según afirma, la enseñanza de lenguas en Colombia necesita una reformulación apremiante de los paradigmas que han determinado sus construcciones culturales, sociales y políticas.

Ante este panorama de las lenguas extranjeras en Colombia y los acercamientos políticos y curriculares que se han hecho sobre educación bilingüe, vale la pena realizar una aproximación histórica y conceptual al marco normativo, la política bilingüe y los lineamientos curriculares de los proyectos educativos derivados del PNB; en concreto, el Programa Bogotá Bilingüe (PBB). Tal acercamiento puede permitir hacer una reflexión sobre los contextos y los retos que enfrentan las instituciones educativas distritales. Esta reflexión puede favorecer la toma de decisiones sobre acciones locales y regionales necesarias para superar o aliviar la tensión entre políticas y lineamientos bilingües basados en globalización y estandarización y las realidades de los salones de clase enmarcadas por los intereses y las necesidades de profesores y estudiantes.

\section{Revisión de literatura}

\section{Marco normativo de lenguas extranjeras (inglés) en Colombia}

García y García (2012) sostienen que la coexistencia de una lengua mayoritaria y varias lenguas 
minoritarias ha conducido a la creación de dos tipos de políticas lingüísticas en Colombia. La primera ha buscado normalizar el contacto entre el español, la lengua mayoritaria del país, con las lenguas minoritarias, indígenas y criollas. El segundo grupo, en cambio, ha procurado implementar programas de educación bilingüe para hablantes de una lengua mayoritaria a través de la inserción de una o diversas lenguas extranjeras. Para estos autores, estas políticas no han sido exitosas porque han desconocido la diversidad lingüística del país al limitarse a la promoción del inglés como lengua extranjera; situación que, en últimas, ha mantenido la idea de que las lenguas criollas son deficientes y no contribuyen al desarrollo del país.

Para Moreno (2009), las políticas educativas en torno a lenguas extranjeras han estado asociadas al logro de mejores niveles de calidad académica y el impulso del desarrollo profesional del país. Como resultado, Moreno sostiene que el marco normativo sobre la enseñanza y el aprendizaje ha estado circunscrito principalmente por discursos de inserción global, productividad y competitividad. Desde la Ley General de Educación de 1994, pasando por planes nacionales y sectoriales y el PNB (tabla 1), el marco normativo ha buscado impulsar la adquisición de habilidades comunicativas en, al menos, una lengua extranjera a lo largo de todo el sistema educativo nacional. Como resultado de este marco, el Estado colombiano ha centrado sus esfuerzos en el mejoramiento del nivel de lengua extranjera mediante cuatro estrategias básicas: desarrollo de estándares, evaluación de competencias, programas de capacitación docente y uso de tecnologías de la información y la comunicación.

\section{Tabla 1}

\section{Marco normativo colombiano sobre enseñanza de lenguas extranjeras}

\begin{tabular}{|l|l|}
\hline $\begin{array}{l}\text { Ley General de } \\
\text { Educación, 1994 }\end{array}$ & $\begin{array}{l}\text { Artículo 21. Objetivo m (ciclo de básica primaria). } \\
\text { Artículo 22. Objetivol (ciclo de básica secundaria). } \\
\text { Artículo 23. Área obligatoria y fundamental 7. }\end{array}$ \\
\hline $\begin{array}{l}\text { Plan Nacional } \\
\text { Decenal de } \\
\text { Educación }\end{array}$ & $\begin{array}{l}\text { Lineamientos para la educación preescolar, básica y media: currículo objetivo 1 y metas 1. } \\
\text { Lineamientos para la educación superior: meta de currículos pertinente, meta en formación y } \\
\text { desarrollo profesional y meta en formación de los docentes de educación superior. }\end{array}$ \\
\hline $\begin{array}{l}\text { Programa Nacional } \\
\text { de Bilingüismo } \\
\text { 2004-2019 }\end{array}$ & $\begin{array}{l}\text { Formulación, publicación y socialización “Estándares básicos de competencia en lenguas ex- } \\
\text { tranjeras: Inglés" (2005-2008) } \\
\text { Sistema Nacional de Evaluación (Inglés Pro-saber). } \\
\text { Formación docente (cursos de inmersión, cursos virtuales de inglés y cursos de mejoramiento). } \\
\text { Usos de medios y nuevas tecnologías (Colombia Aprende). }\end{array}$ \\
\hline $\begin{array}{l}\text { Ley 1651- Ley de } \\
\text { bilingüismo }\end{array}$ & $\begin{array}{l}\text { Adiciones y modificaciones a artículos de la Ley 115 para favorecer el desarrollo de habilidades de } \\
\text { conversación, lectura y escritura al menos en una lengua extranjera. }\end{array}$ \\
$\begin{array}{l}\text { El financiamiento de los costos de la implementación de la ley y la reglamentación del fomento } \\
\text { indigenas y tribales. }\end{array}$ \\
\hline
\end{tabular}

Marco normativo de lenguas extranjeras (inglés) en Bogotá. 
En los últimos años, la enseñanza-aprendizaje de lenguas extranjeras, especialmente inglés, ha sido centro de gran interés del sector público, privado y productivo en Bogotá. En junio de 2001 una reunión entre el Ministerio de Comercio Exterior, la Gobernación de Cundinamarca, la Alcaldía Mayor de Bogotá, el Comité Asesor Regional de Comercio Exterior de Bogotá y Cundinamarca (CARCE) y la Cámara de Comercio de Bogotá propuso emprender acciones a favor de la educación bilingüe en la región mediante la firma de un Acta de Compromiso. En 2003 estas entidades firmaron un Acuerdo de Voluntades para el lanzamiento del Proyecto Bogotá y Cundinamarca Bilingües en Diez Años, cuyo fin era proporcionar herramientas que permitieran la consecución del nivel intermedio. Dentro de este contexto y a la luz del marco normativo del PNB, la SED Bogotá estableció el Programa de Transición hacia Bilingüismo 2004-2019, el cual buscaba garantizar la competencia comunicativa bilingüe de estudiantes y docentes de la ciudad. Como parte de este programa, la Secretaría de Educación, junto con el Consejo Británico seleccionó un grupo de mega colegios para aplicar pruebas piloto del Programa (Pal, 2010).

Por otra parte, un rastreo de los planes sectoriales de educación del Distrito permite hacer un seguimiento del trabajo hecho por las últimas administraciones de la ciudad en cuanto a educación bilingüe. En el Plan Sectorial de Educación 2004-2008 “Bogotá: una gran escuela para que niños, niñas y jóvenes aprendan más y mejor", la alcaldía de Luis Eduardo Garzón estableció el proyecto "Fortalecimiento de una segunda lengua" como parte del programa 8 "Articulación educativa de Bogotá con la región central". Este proyecto buscaba fortalecer el aprendizaje de inglés y otros idiomas mediante actualización metodológica y provisión de recursos especializados. A través del Acuerdo 253 de 2006, el Consejo de Bogotá institucionalizó el Programa "Bogotá Bilingüe". Este acuerdo propuso unos objetivos, unos sectores estratégicos y unas líneas de acción que deberían ser gestionadas por un comité interinstitucional con el fin de formar ciudadanos capaces de comunicarse y desenvolverse en la economía global.

Más tarde, en el Plan Sectorial de Educación 2008-2012 "Educación de calidad para una Bogotá Positiva", la alcaldía de Samuel Moreno estableció que el escaso dominio de una lengua extranjera era un problema que se debía resolver como prioridad de la política educativa de la ciudad. Para tal fin y en pro de una transformación pedagógica de calidad, se creó la acción denominada "Intensificación de la enseñanza de inglés". Como parte de esta acción, la Alcaldía estipuló, entre otras cosas, promover la asignación de mayor número de horas en educación básica y media, dotar con textos y con el software English Discovery, e incentivar a los docentes de inglés a obtener certificados en B-2.

Finalmente, en el Plan Sectorial de Educación 2012-2016 Bogotá Humana, de la alcaldía de Gustavo Petro, se hacen algunas alusiones a segunda lengua en los Programas "Construcción de saberes, educación incluyente, diversa y de calidad" y Programa "Jornada Única". Estas menciones se hacen como parte de una reorganización de la oferta curricular con el fin de intensificar el aprendizaje no solo de una segunda lengua sino de lenguaje, matemática, ciencias naturales y sociales. La única referencia directa al Plan sobre lenguas extranjeras se hace en el objetivo de impacto número 2 del mejoramiento de la educación formal, el cual menciona el establecimiento de 100 colegios con centros de idiomas e intensificación del aprendizaje de una segunda lengua desde preescolar. (Ver el resumen de las propuestas oficiales hechas en Bogotá en la tabla 2).

La revisión, tanto del marco normativo nacional como del marco normativo local, permite entrever que la mayoría de las reformas educativas tienden a señalarles a las instituciones y a los docentes qué y cómo hacer las cosas (visión técnica) en vez de facilitar un conocimiento profundo y de una intervención decidida en contextos educativos (visión reflexiva). Al respecto, Vieira y Moreira 
Tabla 2

\section{Marco normativo bogotano sobre lenguas extranjeras (inglés)}

\begin{tabular}{|c|c|}
\hline Acta de Compromiso de 2001 & Plan de acción para el logro de una ciudad-región bilingüe. \\
\hline Acuerdo de Voluntades de 2003 & Proyecto Bogotá y Cundinamarca bilingües en diez años. \\
\hline $\begin{array}{l}\text { Convenio SED Bogotá y Consejo } \\
\text { Británico }\end{array}$ & Programa de Transición hacia Bilingüismo 2004-2019. \\
\hline $\begin{array}{l}\text { Plan Sectorial de Educación } \\
\text { 2004-2008 "Bogotá: una gran } \\
\text { escuela para que niños, niñas y } \\
\text { jóvenes aprendan más y mejor" }\end{array}$ & $\begin{array}{l}\text { Programa } 8 \text { “Articulación educativa de Bogotá con la región central. } \\
\text { Proyecto “Fortalecimiento de una segunda lengua”. } \\
\text { Acciones: actualización metodológica y provisión de recursos especializados. }\end{array}$ \\
\hline $\begin{array}{l}\text { Acuerdo } 253 \text { de } 2006, \\
\text { "Programa Bogotá bilingüe", } \\
\text { Consejo de Bogotá }\end{array}$ & $\begin{array}{l}\text { Estrategia de trabajo concertada entre distintos estamentos públicos y privados. } \\
\text { Creación de objetivos, líneas de acción y un comité gestor interinstitucional. }\end{array}$ \\
\hline $\begin{array}{l}\text { Plan Sectorial de Educación } \\
\text { 2008-2012 "Educación de cali- } \\
\text { dad para una Bogotá Positiva" }\end{array}$ & $\begin{array}{l}\text { Problema por resolver: escaso dominio de una lengua extranjera. } \\
\text { Acción: intensificación de la enseñanza de inglés. } \\
\text { Estrategias: mayor número de horas de clase, dotación de textos y software, proyec- } \\
\text { tos de inmersión total, certificación B2 para docentes, etc. }\end{array}$ \\
\hline $\begin{array}{l}\text { Plan Sectorial de Educación } \\
\text { 2012-2016 "Bogotá Humana" }\end{array}$ & $\begin{array}{l}\text { Problema: escasa calidad del servicio educativo debido a una pobre inclusión de } \\
\text { educación ambiental, segunda lengua, arte y deporte. } \\
\text { Acción: programa construcción de saberes - Educación incluyente, diversa y de } \\
\text { calidad para disfrutar y aprender. } \\
\text { Objetivo: mejorar los resultados de la educación formal. } \\
\text { Meta de producto: } 100 \text { colegios con centros de idiomas e intensificación del apren- } \\
\text { dizaje de una segunda lengua desde preescolar. } \\
\text { Proyecto: jornada educativa de } 40 \text { horas semanales para la excelencia académica y } \\
\text { la formación integral,y jornadas únicas. }\end{array}$ \\
\hline
\end{tabular}

(2008) sostienen que una perspectiva instrumentalista plantea constricciones a la reflectividad, la autenticidad, la interacción dialógica, la apertura a la innovación y la autonomía. Para Vieira y Moreira, los docentes y sus instituciones pueden ser actores críticos y propositivos si no se les limita a ser consumidores de conocimiento y operadores de mecanismos, sino que se les configura como participantes informados sobre el mejoramiento de las situaciones educativas que experimentan. A continuación se hace un acercamiento más detallado a las políticas lingüísticas y los lineamientos curriculares del Programa Bogotá Bilingüe (PBB).

\section{Políticas lingüísticas y lineamientos curriculares sobre bilingüismo en Bogotá}

Después de analizar las políticas y prácticas educativas, lingüísticas y culturales presentes en tres tipos de colegios bilingües (inglés-español), De Mejía, Ordóñez y Fonseca (2006) señalan una tensión derivada del énfasis que se tiende a dar a posiciones extranjeras pedagógica, lingüística y culturalmente sobre la formación de arraigo e identidad lingüística y cultural que se espera en estudiantes colombianos. Esta tensión sugirió posiciones conflictivas entre concepciones sobre "lo nuestro", "lo extranjero", "integración", "separación", las cuales 
parecían manifestarse en orientaciones y prácticas que trivializaban el concepto de bilingüismo. La presencia de tales tensiones, según concluyeron las autoras, señalaba una despreocupación o un desconocimiento sobre la articulación curricular en la formación tanto en lengua materna como en lengua extranjera.

A partir del anterior estudio, De Mejía y Fonseca (2008) elaboraron un documento que buscaba proporcionar orientaciones para guiar procesos educativos bilingües y multilingües en lenguas extranjeras. En general, se sugirieron orientaciones teóricas que buscaban permitir a colegios interesados poder tomar decisiones sobre cambios, ajustes y mejoras a los procesos curriculares y las prácticas institucionales en pro del desarrollo de programas bilingües. Tales orientaciones se plantearon en términos de indicaciones lingüísticas, culturales, pedagógicas y teóricas que ofrecieran una base para el diseño y la implementación de programas bilingües (tabla 3).

\section{Tabla 3}

\section{Lineamientos para el diseño de programas de educación bilingüe en Colombia}

\begin{tabular}{|c|c|}
\hline $\begin{array}{l}\text { Desarrollo } \\
\text { lingüístico y } \\
\text { académico } \\
\text { bilingüe o } \\
\text { multilingüe }\end{array}$ & $\begin{array}{l}\text { Precisar definiciones sobre el tipo de bilingüismo que se incorpora en el currículo para que sean coherentes con } \\
\text { el perfil del egresado. } \\
\text { Reflexionar acerca de cómo articular el uso de dos o más lenguas en el currículo y en el desarrollo académico. } \\
\text { Velar por la eficiencia y eficacia de las metodologías y estrategias empleadas en el proceso educativo bilingüe. } \\
\text { Establecer una coherencia curricular longitudinal y transversal con relación al desarrollo de dos o más lenguas } \\
\text { en el currículo. } \\
\text { Formular una política lingüística institucional para propiciar el desarrollo armónico de todas las lenguas usadas } \\
\text { en el currículo. } \\
\text { Evaluar la factibilidad de iniciar procesos de bilingüismo desde temprana edad o desde una edad más tardía. } \\
\text { Tener en cuenta la noción cambiante de ‘hablante nativo` para ajustar la evaluación de la proficiencia bilingüe } \\
\text { de los estudiantes. } \\
\text { Crear un ambiente bilingüe a nivel institucional. } \\
\text { Asegurar coherencia entre los lineamientos teóricos que guían los procesos bilingües y las prácticas pedagógicas } \\
\text { llevadas a cabo en el aula. } \\
\text { Ayudar a los padres de familia a entender los procesos y las decisiones en cuanto a la metodología adoptada por } \\
\text { el colegio, a la luz del perfil bilingüe institucional y el contexto educativo. }\end{array}$ \\
\hline $\begin{array}{l}\text { Desarrollo } \\
\text { cultural y de } \\
\text { identidad }\end{array}$ & $\begin{array}{l}\text { Desarrollar una política institucional coherente sobre el tratamiento de las relaciones interculturales. } \\
\text { Fomentar entre los estudiantes la reflexión consciente y crítica acerca de las relaciones interculturales. }\end{array}$ \\
\hline $\begin{array}{c}\text { Formación de } \\
\text { profesores para } \\
\text { la educación } \\
\text { bilingüe }\end{array}$ & $\begin{array}{l}\text { Hacer énfasis en el papel de los profesores bilingües como modelos para imitar role models lingüísticos y cultura- } \\
\text { les para los estudiantes. } \\
\text { Extender la formación para el bilingüismo y la interculturalidad a los docentes de la primera lengua. } \\
\text { Avanzar en la comprensión de los principios básicos de la educación bilingüe y el desarrollo bilingüe. } \\
\text { Propiciar procesos de empoderamiento y apropiación de cambio. }\end{array}$ \\
\hline $\begin{array}{l}\text { Fundamentos } \\
\text { teóricos de } \\
\text { la educación } \\
\text { bilingüe }\end{array}$ & $\begin{array}{l}\text { Definiciones de bilingüismo y educación bilingüe. } \\
\text { Fundamentos del desarrollo de diferentes modalidades de educación bilingüe (modelos de mantenimiento y } \\
\text { enriquecimiento, programas de inmersión). } \\
\text { Factores que inciden en el proceso de desarrollo bilingüe (desarrollo de la lengua materna, bialfabetismo o lec- } \\
\text { tura y escritura en dos lenguas, desarrollo lingüístico y desarrollo académico bilingüe, cambio de código, input y } \\
\text { output lingüistico, bilingüismo temprano vs. bilingüismo tardio). } \\
\text { Metodologías bilingües (Preview-view-review, enseñanza en equipo, evaluación de lengua y contenido). } \\
\text { Aspectos sociales y culturales para una educación bilingüe intercultural (rol de los padres de familia, interculturalidad). }\end{array}$ \\
\hline
\end{tabular}


Al hablar sobre las necesidades para la implementación de un programa bilingüe en colegios distritales, López, Peña, De Mejía, Fonseca y Guzmán (2009) sostienen que se debe tener en cuenta una serie de aspectos que influyen en el uso asociado tanto de lengua materna como lengua extranjera como medios de instrucción: organización y gestión, recursos humanos y físicos, concepción y creencias hacia el bilingüismo, espacios de promoción del bilingüismo (iniciativas y apoyo), tipos de interacción y problemas y necesidades en la transición hacia el bilingüismo (tabla 4). La consideración cuidadosa de tales aspectos por parte de la comunidad educativa puede permitir, afirman estos autores, establecer necesidades que los colegios distritales deben conocer y poder resolver institucional, pedagógica y operativamente. En consecuencia, antes de embarcarse en proyectos de enriquecimiento y fortalecimiento de lenguas, las comunidades educativas deben examinar el grado o nivel de impacto de los aspectos antes descritos según sus condiciones, necesidades y posibilidades. El análisis de estos aspectos permitirá a los colegios de Bogotá, en últimas, diseñar directrices y estrategias que guíen los proyectos bilingües de las instituciones como una comunidad educativa comprometida con procesos y prácticas en $L_{1}$ y L2 tanto de profesores y estudiantes como de directivas, funcionarios y padres de familia.

Con base en los aspectos que se deben considerar para la implementación de programas bilingües, López et al. (2009) también formulan una serie de recomendaciones y orientaciones que se pueden configurar como políticas para establecimientos públicos del Distrito. Concretamente, se ofrecen políticas sobre cuatro dimensiones: organización y gestión, bilingüismo a través del currículo, ambientes de promoción del bilingüismo y recursos materiales e infraestructura (tabla 5). Tales políticas buscan ayudar a directivas, docentes y padres de familia a dinamizar y materializar el proceso de educación bilingüe mediante acciones que guien los procesos y las prácticas institucionales en y fuera del aula de clases y del plantel mismo. Por otra parte, estas políticas procuran orientar a las comunidades educativas a priorizar sus necesidades $y$ encontrar los insumos que requiere diseñar e implementar un programa bilingüe acorde con sus intereses y capacidades.

Tabla 4

Aspectos que influyen la implementación de programas bilingües en colegios distritales

\begin{tabular}{|l|l|}
\hline \multicolumn{1}{|c|}{ Aspecto } & \multicolumn{1}{|c|}{ Definición } \\
\hline Organización y gestión & $\begin{array}{l}\text { Conjunto de acciones, estructura y políticas que planifican, facilitan y coordinan las acti- } \\
\text { vidades de enseñanza-aprendizaje bilingüe de manera global y estratégica. }\end{array}$ \\
\hline Recursos humanos y físicos & $\begin{array}{l}\text { Personal y materiales con que cuenta la institución para planear y gestionar objetivos } \\
\text { comunes y propósitos pedagógicos de un programa bilingüe. }\end{array}$ \\
\hline $\begin{array}{l}\text { Concepciones y creencias } \\
\text { sobre el bilingüismo }\end{array}$ & $\begin{array}{l}\text { Ideas y percepciones que tienen los distintos miembros de la comunidad educativa acer- } \\
\text { ca del bilingüismo y los roles y las potencialidades de L1 y L2 dentro de un programa } \\
\text { bilingüe. }\end{array}$ \\
\hline $\begin{array}{l}\text { Espacios de promoción del } \\
\text { bilingüismo }\end{array}$ & $\begin{array}{l}\text { Conjunto de acciones, decisiones y oportunidades formales y continuas que impulsan y } \\
\text { fomentan de manera sistemática el contacto oral o escrito con L1 y L2 de toda la comu- } \\
\text { nidad educativa. }\end{array}$ \\
\hline Tipos de interacción & $\begin{array}{l}\text { Formas en que se concibe y se gestiona el uso de L1 y L2 para la comunicación dentro de } \\
\text { la institución. }\end{array}$ \\
\hline $\begin{array}{l}\text { Problemas y necesidades } \\
\text { hacia el bilingüismo }\end{array}$ & $\begin{array}{l}\text { Conjunto de obstáculos que dificultan la implementación de la educación bilingüe y se- } \\
\text { rie de requerimientos que tiene la comunidad para avanzar en el diseño e implementa- } \\
\text { ción de un proyecto bilingüe. }\end{array}$ \\
\hline
\end{tabular}


Tabla 5

Políticas para la implementación de programas bilingües en colegios distritales

\begin{tabular}{|c|c|}
\hline Dimensión & Políticas \\
\hline \multirow{12}{*}{$\begin{array}{l}\text { Organización y } \\
\text { gestión }\end{array}$} & Generales \\
\hline & Definir el programa de bilingüismo como área estratégica. \\
\hline & Asignar la coordinación del programa bilingüe a una cabeza visible que cuente con un grupo interdisciplinario. \\
\hline & Asegurar continuidad de aprendizajes individuales y colectivos sobre la implementación del programa bilingüe. \\
\hline & Organizar el uso flexible de recursos y tiempos para permitir trabajo conjunto. \\
\hline & Construir relaciones sólidas entre la comunidad educativa, extendiéndolas a representantes de la comunidad local. \\
\hline & Profesores \\
\hline & Sustentar formación y cualificación profesoral de docentes de L1 y L2 sobre bilingüismo e interculturalidad. \\
\hline & Consolidar la comprensión de los principios de la educación y el desarrollo bilingüe. \\
\hline & Propiciar procesos de empoderamiento y apropiación de cambio. \\
\hline & Estudiantes \\
\hline & Formular un perfil realista de egresado con referencia a la competencia bilingüe. \\
\hline \multirow{25}{*}{$\begin{array}{c}\text { Bilingüismo } \\
\text { a través del } \\
\text { currículo }\end{array}$} & Generales \\
\hline & Adoptar una modalidad de programa bilingüe de intensificación o inmersión parcial según el contexto y el perfil de egresado. \\
\hline & Asegurar el desarrollo articulado de L1 y L2 en el currículo que favorezca el desarrollo tanto comunicativo como académico en las dos lenguas. \\
\hline & Trabajar el bilingüismo como eje transversal e integrador del currículo. \\
\hline & Dimensión intercultural e interlingüistica \\
\hline & Educar a la comunidad sobre el trabajo consciente de la dimensión intercultural de la formación bilingüe. \\
\hline & Tomar conciencia de supuestos culturales de la comunidad educativa. \\
\hline & Leer críticamente discursos pedagógicos sobre cultura e idiomas. \\
\hline & Orientar prácticas formativas en L1 y L2 desde sus aspectos culturales y sus manifestaciones en los sistemas de códigos. \\
\hline & Procesos de lectura y escritura desde el bialfabetismo \\
\hline & Considerar la lectura-escritura desde una visión comunicativa del lenguaje y procesos de enseñanza-aprendizaje acordes. \\
\hline & Promover el bialfabetismo como hecho social desde la cotidianidad de la familia, la comunidad, la institución y los pares. \\
\hline & Hacer énfasis en procesos de lecto-escritura en L1 en los primeros años de escolaridad para favorecer posteriormente la transferencia en L2. \\
\hline & Incentivar usos de recursos físicos y experiencias representativas a través de proyectos que promuevan procesos de lecto-escritura en L1 y L2. \\
\hline & Trabajo interáreas \\
\hline & Enseñar L2 mediante contenidos que favorezcan el desarrollo lingüístico y académico. \\
\hline & Integrar la enseñanza de L2 a un contenido al nivel cognitivo del estudiante. \\
\hline & Escoger contenidos de acuerdo con las necesidades y expectativas de los estudiantes. \\
\hline & Asegurar el trabajo interdisciplinario y colaborativo de contenidos y actividades que desarrollen las habilidades lingüísticas y académicas de los estudiantes. \\
\hline & Evaluación formativa y permanente \\
\hline & Evaluar continua y diversamente el alcance de los objetivos de enseñanza-aprendizaje y la competencia comunicativa de los estudiantes. \\
\hline & Informar a los estudiantes sobre los objetivos de las evaluaciones. \\
\hline & Usar evaluaciones de desempeño que midan auténticamente la competencia de los estudiantes. \\
\hline & Alinear la evaluación a la instrucción con fundamento en los estándares básicos de competencias en lenguas extranjeras. \\
\hline & Capacitar a los docentes para diseñar, adaptar, usar e interpretar evaluaciones formativas y permanentes en el salón de clases. \\
\hline \multirow{4}{*}{$\begin{array}{l}\text { Ambientes de } \\
\text { promoción del } \\
\text { bilingüismo }\end{array}$} & Valorar las experiencias de los estudiantes. \\
\hline & Asignar el mismo estatus al desarrollo de L1 y L2 y fomentar el uso de las dos lenguas en la institución. \\
\hline & Crear espacios para recibir input comprensible y para interactuar comunicativamente en L2. \\
\hline & Involucrar a toda la comunidad en el proceso de bilingüismo mediante talleres o charlas. \\
\hline \multirow{3}{*}{$\begin{array}{c}\text { Recursos } \\
\text { materiales e } \\
\text { infraestructura }\end{array}$} & Crear salones en donde se fomente el uso y el desarrollo de L1 y L2 a través de materiales auténticos y recursos didácticos. \\
\hline & Crear espacios para la práctica y el refuerzo de L1 y L2 fuera del salón de clases, en especial a través de centros de recursos de idiomas (CRI). \\
\hline & Dotar la biblioteca de materiales apropiados en L1 y L2. \\
\hline
\end{tabular}


Como resultado de esta aproximación a políticas y lineamientos relacionados con el PBB es posible evidenciar un esfuerzo por incorporar la formación bilingüe en el funcionamiento institucional y los procesos formativos de las instituciones educativas distritales. Esta incorporación se ha hecho mayoritariamente a través del establecimiento de una lengua extranjera en el currículo escolar desde temprana edad. Incluso se ha propiciado la enseñanza de otras asignaturas en la lengua extranjera. Al respecto, Galindo y Moreno (2008) sostienen que la legislación de la formación bilingüe escolar en Colombia se ha cimentado mayoritariamente en lograr una adecuada apreciación de la estructura lingüistica, una mejor capacidad comunicativa y una valoración cultural superior. Sin embargo, señalan que el éxito de esta legislación demanda tanto la participación de los actores del tejido sociopolítico de las instituciones educativas como el abordaje multidimensional desde principios del bilingüismo y la didáctica de las lenguas extranjeras. Las realidades y las necesidades de las instituciones educativas distritales que se analizan a continuación parecen sugerir que las acciones y las decisiones oficiales en Bogotá han dejado de lado tanto la participación como el abordaje para centrarse en la estipulación de direccionamientos para el funcionamiento institucional y el desempeño docente.

\section{Proyectos bilingües en colegios de Bogotá: realidades y necesidades}

El establecimiento de fases para proyectos de educación bilingüe y la especificación de un conjunto de políticas de diseño e implementación no se constituyen en medidas ni acciones suficientes. Tales iniciativas son necesarias pero son de carácter básico o inicial; es decir, ayudan a comenzar el proceso de educación bilingüe pero no garantizan el éxito de su desarrollo. Además del establecimiento de políticas y estrategias, es importante que tanto la SED Bogotá como las instituciones educativas distritales reconozcan la complejidad de la realidad tanto en el interior como en el exterior de las comunidades educativas de la ciudad.

Esta realidad desempeña un papel predominante en el éxito del enriquecimiento o fortalecimiento de L1 y L2 porque determina el nivel de respuesta del plantel y la comunidad ante las exigencias curriculares, pedagógicas e institucionales que conlleva la educación bilingüe. Por otra parte, esta realidad impacta la continuidad y la articulación de las decisiones y los procesos a los que se deben comprometer todos los actores del proceso; es decir, el Ministerio de Educación, Secretarías de Educación, directivas, profesores, estudiantes y padres de familia.

En cuanto a la realidad y las necesidades de proyectos bilingües en Bogotá y teniendo en cuenta que a través de los años se han propuesto medidas y programas para superar las limitaciones del aprendizaje de lenguas extranjeras y cualificar su enseñanza, Pal (2010) sostiene que es pertinente ahondar en las dimensiones académicas y culturales de la lengua de manera que se trascienda discursos y prácticas encaminados al desarrollo comunicativo solicitado hoy por el mundo laboral. Para ella, tanto el Ministerio de Educación Nacional (MEN) como la SED Bogotá deberían replantear propósitos y objetivos que les permitan tanto a estudiantes como a profesores trabajar con competencias orientadas a la comprensión de otras formas de vida y la ampliación de las perspectivas de realización. Por otra parte, aunque se evidencia cierta diversidad y recursividad en el tipo de actividades empleadas en la clase de inglés, especialmente prácticas relacionadas con la lúdica, la música y la lectura, Pal indica que estas acciones parecen no responder a secuencias didácticas sólidas que procuren suscitar experiencias formativas enriquecedoras con base en las necesidades y condiciones de aprendizaje y los propósitos de la educación bilingüe.

Igualmente, $\mathrm{Pal}$ (2010) afirma que los proyectos de bilingüismo deben tener en cuenta no solo la en- 
señanza de los contenidos de la disciplina como tal sino también la interacción articulada con contenidos de otras áreas del currículo y el contexto real de los estudiantes. De esta manera, Pal argumenta que los proyectos bilingües en desarrollo deben impulsar más y mejor la transversalidad curricular para trascender un trabajo que suele limitarse a cubrir lenguaje del aula de clases, instrucciones y vocabulario clave. Finalmente, Pal señala que es necesario incluir a los padres en la enseñanza de inglés de manera que ellos no solo puedan colaborar con tareas asignadas sino también llevar un proceso coherente con las directrices de un programa bilingüe.

Por su parte, López, Peña y Guzmán (2011) sostienen que los procesos de transición hacia el bilingüismo en colegios públicos tienen requisitos especiales debido a las directrices educativas que los rigen, la diversidad y la cantidad de su población y los rasgos de su planta docente. Por ello, recomiendan estos autores hacer una revisión cuidadosa del contexto, los procesos y los actores que permita asegurar la estabilidad y la perdurabilidad de las iniciativas que se tomen por la SED Bogotá o los planteles mismos. Al referirse a las necesidades para hacer la transición hacia el bilingüismo, López, Peña y Guzmán hablan de:

- Disponer de mayores recursos acordes con la edad y el nivel de los estudiantes, que puedan utilizarse dentro y fuera de la institución.

- Ofrecer formación profesional a los profesores de manera continua y sistemática para trascender una simple formación lingüística y adentrarse en pedagogías bilingües que permitan diálogos inter-tras-culturales y pensamiento investigativo.

- Buscar estrategias que fomenten la interacción y el uso de lengua extranjera en el salón de clases sin dejar de lado el español como lengua materna.
- Integrar a los padres de familia y la comunidad para que se garantice su participación activa en el proceso de bilingüismo.

Por otra parte, Peña, Flaborea y Guzmán (2011) encontraron una desconexión entre la existencia de ciertas políticas lingüísticas institucionales y la carencia de planes estructurados de implementación. Igualmente, estos autores observaron la existencia de algunas problemáticas relacionadas con los docentes, los estudiantes y los padres de familia sin discusión ni acercamiento suficientes. Para Peña, Flaborea y Guzmán, los planteles educativos deben poder y saber transformarse en agentes de cambio, capaces de hacer revisiones a su malla curricular y crear planes de acción para el crecimiento institucional. Para que dicha transformación sea factible, los autores recomiendan satisfacer necesidades como:

- La construcción de un currículo formal que de manera clara y sólida dé cuenta de la dirección, los propósitos, las metodologías, las formas de evaluación y los materiales de instrucción.

- La integración del área de español e inglés con el fin de crear ambientes de aprendizaje que fomenten el uso institucional y el crecimiento cultural de las dos lenguas.

- La creación de procesos continuos de reflexión pedagógica y autoevaluación a través de espacios de trabajo interdisciplinario.

- El involucramiento activo y entusiasta de los estudiantes como coarquitectos de los contenidos y las prácticas bilingües a través de proyectos de tipo académico y cultural en el aula y fuera de esta.

La revisión de las realidades y las necesidades del PBB sugiere que las directrices y gestiones tanto de la Alcaldía como de la SED Bogotá se han centrado mayoritariamente en el inglés como lengua extranjera. Tales acciones se han preocupado por regular los procesos y las prácticas de las instituciones, los profesores y los estudiantes de cole- 
gios de primaria y secundaria. Sus metas parecen haber sido fundamentalmente dos: el desarrollo del nivel de lengua y el mejoramiento del proceso de enseñanza-aprendizaje. Vale la pena destacar el hecho de que las acciones se han iniciado y diseñado desde dos grandes marcos de referencia: política lingüistica y lineamientos curriculares. Estos marcos, sin embargo, se han trabajado de manera dispersa y desarticulada sin lograr precisar de manera clara y puntual su relación con la realidad escolar de las instituciones educativas distritales. Esta situación no solo ha delimitado la naturaleza y el propósito de los proyectos de bilingüismo en la ciudad sino que da lugar a tensiones o desencuentros entre decisiones oficiales de corte homogeneizador y contextos educativos de naturaleza heterogénea. Ver a continuación la discusión al respecto.

\section{Bogotá Bilingüe: tensión entre política lingüística, lineamientos curriculares y realidad escolar}

Hablar de política lingüística exige analizar la ideología lingüística (sistema cultural de objetivos y nociones sobre la naturaleza de la lengua y sus relaciones lingüísticas y sociales), las prácticas lingüísticas (comportamientos lingüísticos observables y aceptables en una comunidad) y la planeación lingüística (intervenciones y esfuerzos planeados para modificar prácticas y creencias lingüísticas) que determinan el uso y el aprendizaje de lenguas (Spolsky, 2004). Desde la perspectiva de Spolsky, es probable hacer un análisis básico de la política lingüística que se ha configurado a través del PBB. En primera medida, este programa ha buscado delinear las prácticas lingüísticas de profesores y estudiantes con base en el documento "Estándares básicos de competencias en lenguas extranjeras: inglés", documento encaminado hacia un alfabetismo funcional en lengua extranjera según las demandas del mercado (Vargas, Tejada y Colmenares, 2008). Por otra parte, la ideología lingüística del programa ha estado marcada por una visión instrumentalista orientada hacia nocio- nes como el trabajo, la globalización y la competitividad, las cuales han propiciado la estandarización, la certificación y la estratificación del inglés (Usma, 2009). Finalmente, la planeación lingüística de Bogotá Bilingüe, al igual que la del PNB, representa un caso manifiesto de programación de adquisición de lengua enfocado mayoritariamente a proveer oportunidades en el sistema educativo que permitan elevar el conocimiento del inglés de los estudiantes de primaria y secundaria (Herazo, Jerez y Lorduy, 2012). En resumen, la política lingüística del PBB se caracteriza por el funcionalismo, la instrumentalización y la programación, rasgos que determinan su naturaleza y constriñen el alcance de las acciones que instituciones y comunidades educativas pueden emprender.

Por su parte, Shohamy (2009) afirma que la política lingüística debe estudiar también los mecanismos o dispositivos que se emplean para alterar o perpetuar determinados comportamientos lingüísticos según ciertas agendas. Para Shohamy, mecanismos como regulaciones educativas, exámenes de lengua y evaluación docente no solo deberían imponer ideologías y crear prácticas lingüísticas sino que deberían posibilitar la negociación entre diferentes actores. En otras palabras, estos dispositivos deberían poder ofrecer un acercamiento abierto, dialógico y constante a las decisiones y las prácticas lingüísticas según las realidades y dinámicas de las comunidades e instituciones. Sin embargo, un acercamiento a los mecanismos del PBB permite reconocer, entre otras cosas, que la política lingüística del Distrito Capital se ha movido más al vaivén de las agendas gubernamentales y los discursos internacionales que de la participación activa de las comunidades educativas de la ciudad, en especial de los mismos profesores y estudiantes a quienes se espera involucrar y beneficiar. Las normas y regulaciones sobre cuál lengua extranjera aprender y cómo y cuándo enseñarla reflejan decisiones verticales que, en gran parte, solo imponen metas, métodos y procesos sin tener en cuenta los contextos reales de los colegios públicos. 
La falta de diálogo y el desconocimiento del contexto van en contravía de lo que los expertos aconsejan en cuanto a la implementación de políticas lingüísticas educativas (Nunan, 2003).

Al hablar de política lingüística, Kroon (2000) sostiene que esta debe ser resultado de una actividad sistemática y propositiva encaminada a lograr metas bien definidas mediante medios y cronogramas puntuales. Para su éxito, la política debe poder generar tanto acuerdos ideológicos como agendas temáticas compartidas sobre el asunto central de la política. Tales acuerdos permiten preparar y dar forma a contenidos y acciones que posteriormente se implementarán y evaluarán de manera estructurada. Una vez evaluada, la política puede ser objeto de reformulaciones, rediseños o ajustes que lleven a su feliz término. Sin embargo, es perceptible que la política lingüística del PBB se ha desarrollado de una manera prescriptiva e instrumentalista. En gran medida, las entidades encargadas de la política la han formulado e implementado de manera vertical y se han preocupado esencialmente por hacer un seguimiento y una evaluación enmarcados en la rendición de cuentas y la eficiencia a través de cifras, porcentajes y promedios. Por otra parte, esta política no ha tenido en cuenta la naturaleza y la dinámica multilingüe y multicultural de nuestra sociedad y del mundo hoy. Su diseño e implementación se han hecho con pocas inclusiones de perspectivas sociocríticas e históricas que den cuenta de la diversidad de intereses e interacciones de los diferentes grupos sociales.

Por otra parte, hablar sobre la noción de lineamientos curriculares es un reto porque el concepto de currículo es polisémico y polimorfo. Una revisión de la literatura arroja múltiples definiciones, diferentes corrientes, posturas teóricas y variadas interpretaciones. Para Malagón (2008), es oportuno contar con un marco de referencia que ayude a caracterizar las diferentes propuestas curriculares. Un posible marco de referencia es la teoría de los intereses de los saberes de Habermas (1990): técnico, práctico y emancipato- rio o crítico. Desde el interés técnico, el diseño curricular busca explicar y describir el mundo mediante el control del ambiente educativo para que el producto que resulte sea acorde con objetivos preestablecidos para la enseñanza y el aprendizaje. Por su parte, el interés práctico le aporta al diseño curricular una preocupación por comprender e interpretar el mundo social y cultural a través de la generación de conocimiento subjetivo basado en el diálogo y la interacción. Finalmente, el diseño curricular basado en el interés emancipatorio o crítico busca trasformar el mundo mediante la generación de autonomía y libertad que permita la consecución de sujetos capaces de realizar autorreflexión y autoentendimiento.

En educación bilingüe, hablar del currículo implica discutir sobre una articulación entre objetivos, modelos y módulos educacionales que busque desarrollar la habilidad para usar dos o más lenguas con facilidad y eficacia (Siguan, 1986). Para Ferguson, Houghton y Well (1977), dicha articulación se puede dar desde tres tipos de educación bilingüe: transaccional, de mantenimiento y de enriquecimiento ${ }^{2}$. Cada tipo es movido por intencionalidades y actividades que van desde la integración social, la conservación cultural o el enriquecimiento lingüístico; factores que a la vez influyen esencialmente en decisiones sobre la metodología. Al respecto, Halbach (2008) afirma que una de las principales características metodológicas de la educación bilingüe debe ser la coordinación entre la enseñanza de lengua y la enseñanza de contenidos. Para Halbach, tal coordinación se facilita cuando se emplea un enfoque temático y experiencial que favorezca la complementariedad entre varias asignaturas al estudiar la misma temática desde diferentes ángulos con el fin de crear unidades memorables y significativas.

2 La educación bilingüe transaccional busca la adquisición de una L2 mayoritaria para conseguir una integración social que L1 no tiene $u$ ofrece. Por su parte, la educación de mantenimiento busca la conservación de lenguas minoritarias al mismo nivel de dominio de una lengua mayoritaria. Finalmente, la educación bilingüe de enriquecimiento busca el desarrollo de una competencia aceptable en una L2 extranjera y minoritaria con relación al uso mayoritario de L1. 
Desde el punto de vista del currículo, tanto el PNB como el PBB son resultado de un interés técnico en cuanto se entiende el currículo como un plan de instrucción concebido de manera universal, ajeno a contextos y sujetos concretos. Es decir, el currículo consiste en la ejecución de un modelo de enseñanza-aprendizaje en el cual los objetivos, los temas y las estrategias de acción están previstos por especialistas para que profesores y estudiantes los implementen en sus salones de clases de manera controlada y eficaz (Ferrández, 1991). Por otra parte, este modelo ve a los profesores como simples operarios educativos, posición que dista mucho de la expresada por autores como Stenhouse (1985), quien aboga por la idea del profesor como investigador de su propia práctica en contexto. Para el PBB, el profesor parece ser un instructor/funcionario que necesita seguir el currículo como una serie de especificaciones lingüísticas y metodológicas programadas para el aula. Aunque hay ciertos enunciados en los documentos sobre empoderamiento y reflexión docente, las prácticas y los procesos tanto de la SED Bogotá como de las mismas instituciones educativas pocas veces permiten la configuración del profesor de lengua extranjera como profesional reflexivo capaz de asumir el currículo como un conjunto de finalidades y prácticas que se deben interpretar, adaptar e, incluso, recrear en la enseñanza.

Por otra parte, el currículo que contempla el PBB parece ser un modelo de educación bilingüe de enriquecimiento que, en gran parte, no pasa de ser una simple serie de lineamientos y orientaciones encaminados a ofrecer puntos de referencia para el análisis o la revisión de los programas académicos de colegios bilingües o de intensificación en lengua extranjera. Es decir, el programa de la ciudad al igual que el programa nacional no cuenta con un diseño curricular que asegure el desarrollo y la integración sistemática de la competencia comunicativa e inter y multicultural tanto en L1 como en L2. Al respecto, Fandiño, Bermúdez y Lugo (2012) sostienen que se deben precisar enfoques y estrategias que permitan hacer un trabajo longitudinal y transversal a lo largo de la formación bilingüe. Tal precisión exige dar atención prioritaria y urgente a tres áreas relacionadas con la formación bilingüe de los estudiantes colombianos: concreción del currículo, cualificación del proceso de enseñanza-aprendizaje de L1 y L2 y fortalecimiento del sentido de comunidad bilingüe. Dicha atención puede empezar con la discusión seria y la implementación cuidadosa de conceptos como bialfabetización, biculturalismo, interculturalidad y aprendizaje integrado entre contenido y lengua extranjera.

Finalmente, el hablar de la realidad escolar de las instituciones educativas distritales conlleva analizar factores contextuales, actores involucrados y oportunidades educativas. Al respecto, Maturana (2011) sostiene que dentro del marco de la enseñanza y el aprendizaje con inglés como lengua extranjera, la realidad de las comunidades e instituciones públicas va de la mano de factores tanto internos como externos que influyen las relaciones que se establecen entre los integrantes centrales de estos procesos: los profesores, los estudiantes y el saber específico. Para Maturana, los factores internos son un conjunto de elementos subjetivos e intersubjetivos que intervienen en los procesos de enseñanza y aprendizaje; concretamente, esta autora habla de actitudes, creencias, percepciones, motivación y estrategias. Estos elementos son susceptibles de modificación o refuerzo a través de la interacción entre los integrantes centrales de estos procesos. Por otra parte, los factores externos son elementos que afectan los procesos de enseñanza y aprendizaje pero que no están dentro del rango de injerencia directa de maestros ni estudiantes. En este aspecto, Maturana señala que situaciones como el tiempo de clase, el acceso y uso de recursos y el desarrollo profesional son variables relevantes en el quehacer de los docentes mientras que el entorno familiar, la cultura y el sistema educativo son variables que inciden de manera particular en el aprendizaje de los estudiantes. 
En una evaluación del PBB, Rodríguez (2011) encontró diez factores que surgieron de la revisión de las condiciones y las dinámicas de los planteles (tabla 6). En el marco de estos factores, Rodríguez sostiene que es importante sopesar e interpretar rasgos contextuales en el momento de diseñar políticas y lineamientos puesto que aspectos como las realidades del uso lingüístico en las comunidades y las necesidades percibidas de los estudiantes determinan el progreso de los procesos de desarrollo bilingüe. Igualmente, Rodríguez señala que se debe tener en cuenta a todos los actores involucrados en la implementación de las políticas bilingües para establecer procedimientos y alternativas apropiadas de ejecución. El análisis del trabajo hecho por la Secretaría de Educación de Bogotá (SED Bogotá) reveló, según Rodríguez, no solo una falta de mecanismos para garantizar la participación y la continuidad de los docentes en los procesos de formación, sino una falta de articulación entre las estrategias de tipo curricular para las instituciones y las de tipo didáctico para las aulas de clases.

\section{Tabla 6}

\section{Factores relevantes en colegios y Secretaría de Educación para el proyecto Bogotá Bilingüe}

\begin{tabular}{|c|c|c|}
\hline \multirow{5}{*}{ Colegios } & $\begin{array}{l}\text { Gestión y } \\
\text { administración }\end{array}$ & $\begin{array}{l}\text { Trabajo planeado y sistemático para la formulación y el desarrollo de proyectos institucionales. } \\
\text { Procesos de comunicación entre los diferentes actores en las instituciones y fuera de estas. } \\
\text { Nivel de conocimiento del proyecto entre docentes, estudiantes y directivas. }\end{array}$ \\
\hline & $\begin{array}{l}\text { Diseño y estructura } \\
\text { curricular }\end{array}$ & $\begin{array}{l}\text { Diseño y estructura de los planes curriculares (definición de contenidos temáticos en la asigna- } \\
\text { tura de inglés, establecimiento de objetivos de aprendizaje, etc.). } \\
\text { Intensidad horaria por semana. } \\
\text { Enseñanza de asignaturas diferentes a inglés en L2. }\end{array}$ \\
\hline & Formación docente & $\begin{array}{l}\text { Preparación académica previa de los docentes. } \\
\text { Planes de cualificación (competencias en L2, metodologías para la enseñanza de L2). }\end{array}$ \\
\hline & $\begin{array}{l}\text { Evaluación y } \\
\text { seguimiento }\end{array}$ & $\begin{array}{l}\text { Estrategias o mecanismos de acompañamiento, seguimiento y verificación de avances. } \\
\text { Estrategias de evaluación del proceso de formación bilingüe. }\end{array}$ \\
\hline & $\begin{array}{c}\text { Recursos (humanos } \\
\text { y físicos) }\end{array}$ & $\begin{array}{l}\text { Talento humano (número de docentes cualificados, nombramiento en propiedad, cargas acadé- } \\
\text { micas, circunstancias personales, trabajo interdisciplinario y colaborativo). } \\
\text { Infraestructura (aula especializada, recursos y materiales de apoyo, bibliotecas). }\end{array}$ \\
\hline \multirow{5}{*}{$\begin{array}{l}\text { Secretaría de } \\
\text { Educación }\end{array}$} & Formación docente & $\begin{array}{l}\text { Aprendizaje del inglés. } \\
\text { Actualización de metodologías de enseñanza. } \\
\text { Creación de centros de recursos especializados y dotación de los existentes. } \\
\text { Intercambios educativos y pedagógicos con la región. } \\
\text { Apoyo de instituciones de carácter gubernamental y privado. }\end{array}$ \\
\hline & $\begin{array}{l}\text { Diseño y estructura } \\
\text { curricular }\end{array}$ & $\begin{array}{l}\text { Divulgación del documento "Estándares básicos de competencias en lenguas extranjeras: inglés". } \\
\text { Diseño y construcción de currículo hacia una educación bilingüe a través de enseñanza por con- } \\
\text { tenidos. } \\
\text { Intensificación de la enseñanza de inglés en primaria y secundaria. } \\
\text { Adopción del modelo de bilingüismo aditivo (L2 se añade al repertorio lingüístico del hablante } \\
\text { para aportar aptitudes cognitivas y sociales). }\end{array}$ \\
\hline & $\begin{array}{l}\text { Gestión y } \\
\text { administración }\end{array}$ & $\begin{array}{l}\text { Acompañamiento institucional para construir propuestas curriculares en colegios pilotos. } \\
\text { Acompañamiento docente en el salón de clases a través de instituciones de educación superior. } \\
\text { Fortalecimiento del inglés con "Talk to the world", curso ofrecido con el apoyo de la Secretaría } \\
\text { Distrital de Desarrollo Económico. } \\
\text { Cursos y especializaciones en metodología y pedagogía bilingüe (Universidad UNINCCA). }\end{array}$ \\
\hline & $\begin{array}{l}\text { Evaluación y } \\
\text { seguimiento }\end{array}$ & $\begin{array}{l}\text { Elaboración de fichas de tipo EBI (Estadística Básica de Inversión) que permiten identificar, justi- } \\
\text { ficar y valorar proyectos públicos. } \\
\text { Revisión a través de las fichas de los planes de acción por parte de entidades gubernamentales } \\
\text { del orden local. } \\
\text { Visitas de funcionarios de SED a colegios pilotos. }\end{array}$ \\
\hline & Recursos & $\begin{array}{l}\text { Proyecto de fortalecimiento de una segunda lengua ( } \$ 6.299 \text { millones) a través de dotación de } \\
\text { centros de recursos de idiomas, certificación de maestros y lineamientos de política. }\end{array}$ \\
\hline
\end{tabular}


Finalmente, es importante anotar que el abordaje de las realidades escolares de los colegios públicos de Bogotá implica examinar la naturaleza de las oportunidades educativas que se ofrecen o posibilitan. Al respecto, y dentro del marco de los programas de bilingüismo, Dávila (2012) explica que los estudiantes de los colegios públicos no solo no cuentan con un conocimiento y dominio satisfactorios del inglés como lengua extranjera en su acervo cultural sino que no tienen oportunidades para movilizarse positivamente en la escala social, situación que los condena a reproducir estados de pobreza cultural y habitus ${ }^{3}$ desfavorables. Por otra parte, señala Dávila que las realidades de las instituciones educativas distritales no garantizan la presencia o la continuidad de factores que son relevantes para el éxito del planteamiento y la ejecución de programas bilingües: dinamismo institucional, capacidad material, renovación pedagógica y centralidad de la lengua extranjera. De esta manera, el problema del bilingüismo en los colegios públicos de Bogotá no radica en la incapacidad de los profesores y de los estudiantes para trabajar con una lengua extranjera, sino en la existencia de una serie de desigualdades que afectan la capacidad de la comunidad educativa para asumir procesos de aprendizajes bilingües como situaciones significativas y útiles para su vida (p. 66).

La tensión derivada de la falta de articulación entre política lingüística, lineamientos curriculares y realidad escolar ha generado un estado de incertidumbre entre los diferentes estamentos y participantes del PBB. Resultado de esta disgregación parece ser la falta de definición de una política y un currículo que respondan de manera puntual a las necesidades y los intereses de la comunidad educa-

3 Para Bourdieu (1972), el habitus se define como un sistema de disposiciones durables y transferibles predispuesto a funcionar como matriz estructurante de las percepciones, las apreciaciones y las acciones de los sujetos (p. 178). Por su parte, Moreno y Ramírez (2003) lo entienden como un conjunto de disposiciones para obrar, sentir, pensar, percibir de una manera determinada de acuerdo con las necesidades de contextos socioculturales determinados y a las exigencias de las instituciones de las que se hace parte (p. 19). tiva de la ciudad. Por otra parte, esta tensión puede haber contribuido a la falta de toma de medidas sistemáticas y críticas en cuanto a investigación, implementación y evaluación que garanticen el alcance del programa.

\section{Consideraciones finales}

Indudablemente, la implementación de comunidades bilingües y multilingües es una ganancia para la mayoría de las sociedades. En el caso del PBB, es visible la existencia de un marco normativo que con base en disposiciones nacionales busca regular las metas, los planes de acción y las estrategias de implementación de instituciones educativas distritales. Este marco se ha preocupado principalmente por procurar fortalecer el aprendizaje de inglés a través de cambios curriculares, actualización metodológica y provisión de recursos especializados. Por otra parte, el Programa cuenta con una serie de orientaciones y lineamientos que buscan ofrecer referentes conceptuales y procedimentales para la implementación de programas bilingües o de fortalecimiento de lengua extranjera. Estas directrices pretenden guiar los procesos y las prácticas de las instituciones y asesorar a las comunidades educativas sobre cómo priorizar sus necesidades para gestionar efectivamente los procesos y los recursos que se requieren. En resumen, Bogotá Bilingüe ofrece normas y directrices que buscan impulsar la formulación y el direccionamiento de proyectos educativos en $\mathrm{L} 2$.

Sin embargo, el PBB presenta una serie de debilidades e imprecisiones que afectan su integralidad y efectividad. Por un lado, el Programa no reconoce la complejidad de la realidad tanto del funcionamiento de los planteles públicos como las dinámicas de las comunidades educativas de Bogotá. Además, deja de lado factores internos y externos que juegan un papel importante en las prácticas y las interacciones de directivas, profesores, estudiantes y padres de familia. Por otra parte, es palpable una falta de precisión metodológica y pedagógica que 
ISSN $0123-1294$ | Educ.Educ. Vol. 17. No. 2 | Mayo-Agosto de 2014 | pp. 215-236.

Universidad de La Sabana | Facultad de Educación

permita el diseño y la realización de experiencias formativas no solo en la enseñanza-aprendizaje de inglés como lengua extranjera sino en la enseñanza-aprendizaje de contenidos a través del inglés. Así mismo, se percibe la falta de estrategias que fomenten la articulación entre L1 y L2 a través de un trabajo transversal e interdisciplinario.

En consecuencia, se hace necesario que se replanteen y redireccionen los marcos de referencia sobre política lingüística y currículo presentes en el PBB. Por una parte, la SED Bogotá y las entidades afines deben superar una política lingüística con un énfasis funcional, instrumentalista y programático, caracterizado por el desconocimiento del contexto y la voz de los actores del proceso. Por otra parte, el diseño curricular de enriquecimiento de L2 debe superar su marcado interés técnico por desarrollar una competencia comunicativa bilingüe sin especificar ni conceptual ni metodológicamente conceptos como bialfabetización, biculturalismo, interculturalidad y aprendizaje integrado entre contenido y lengua extranjera (ver figura 1 sobre la situación del Programa Bogotá Bilingüe). Por su importancia en la reconfiguración de Bogotá Bilingüe y el desarrollo de su marco teórico, la relación entre política lingüística, diseño curricular y realidad escolar es una cuestión que debe abordarse de manera más profunda por la comunidad académica colombiana. Dicho abordaje debe, en últimas, lograr una reconfiguración o reinterpretación del modelo que sustenta el Programa en su totalidad. De esta manera, se favorecería el no caer en acciones y decisiones aisladas al vaivén de los intereses de la administración de turno.

Figura 1. Situación del Programa Bogotá Bilingüe

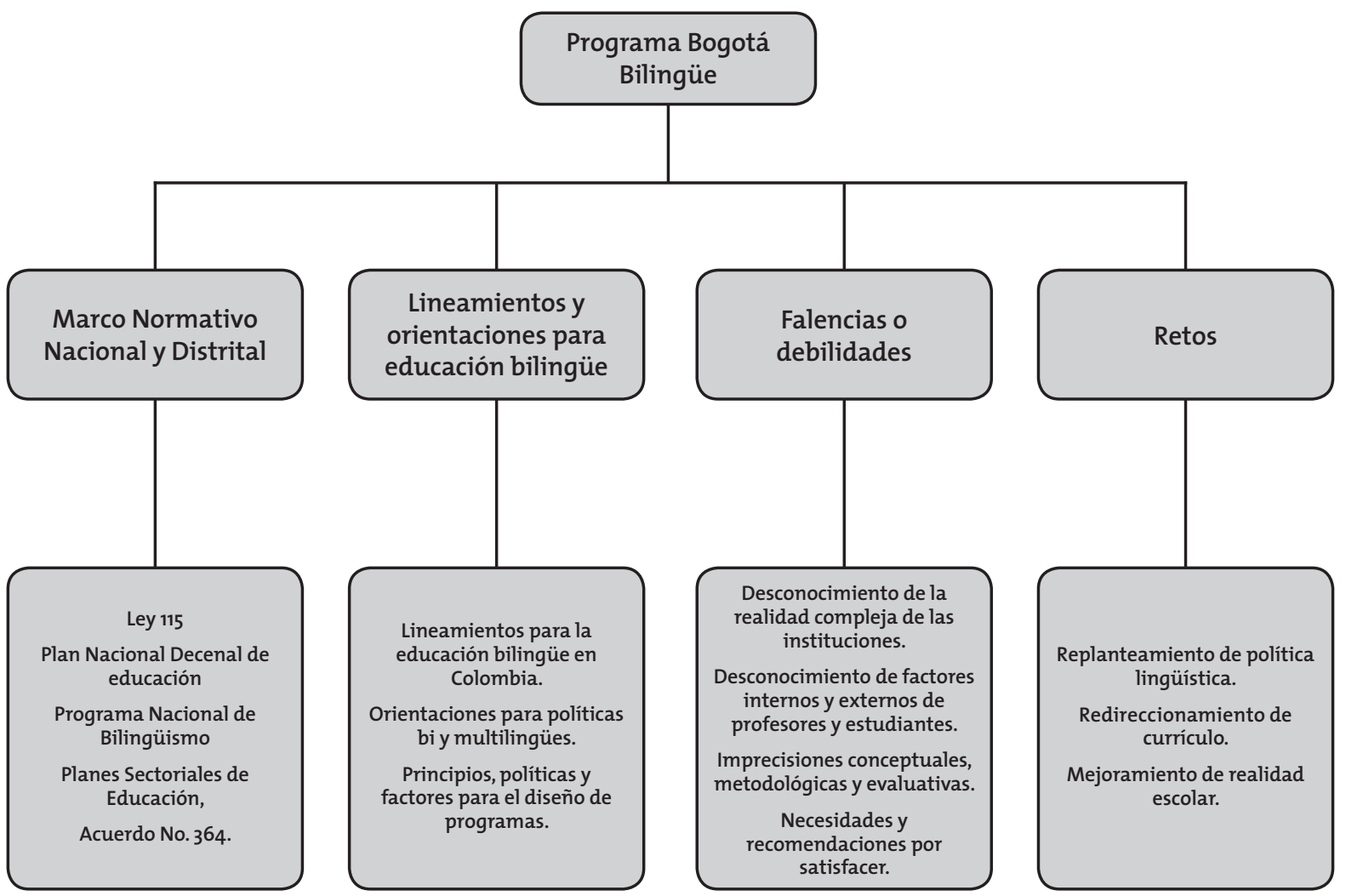


Una posible forma de reconfigurar tanto el PBB como el PNB puede ser el pasar de un modelo burocrático o racionalista de formulación y ejecución de políticas y lineamientos hacia un modelo sociocultural crítico de construcción e implementación. Al respecto, Correa y Usma (2013) afirman que en el modelo burocrático actual las decisiones suelen ser tomadas por los altos niveles de las organizaciones, haciendo caso omiso de antecedentes y condiciones. Este modelo, igualmente, favorece la aceptación de ciertos discursos y supuestos que no permiten formar una imagen más amplia del fenómeno en cuestión. Para contrarrestar estas falencias, Correa y Usma sugieren la adopción de un modelo sociocultural crítico que se basa en discusiones y acuerdos de todas las partes interesadas sobre asuntos como las lenguas que se van a promover, los asuntos que se van a priorizar y las acciones concretas por emprender. Este modelo, en últimas, no busca la homogeneización o estandarización de prácticas y procesos sino que procura que cada comunidad responda de manera pertinente a sus propias situaciones a través de la puesta en marcha de estudios de caso multicéntricos de corte longitudinal y etnográfica.
El anhelo es que este artículo sobre las políticas lingüísticas, los lineamientos curriculares y las realidades escolares pueda iluminar la toma de decisiones que permitan ajustar las acciones, los procedimientos y los mecanismos del PNB y el PBB. Tales mejoras requieren una reconfiguración de las relaciones entre las instituciones educativas, los responsables políticos y las partes interesadas. Igualmente, exigen un compromiso colectivo por trabajar juntos con el fin de garantizar una enseñanza exitosa y un aprendizaje valioso de las lenguas extranjeras. En últimas, se espera que este artículo ayude a redireccionar perspectivas conceptuales, planteamientos formativos y metas institucionales para ver:

el bilingüismo no solo como una manera de preparar a los individuos para las exigencias y necesidades socioeconómicas de un mundo globalizado, sino como la posibilidad de promover hombres y mujeres conscientes de la variedad lingüistica y cultural del mundo, ciudadanos respetuosos del otro e interesados en reconocer y proteger las diferencias y las particularidades de las lenguas y sus culturas (Fandiño, Bermúdez y Lugo, 2012, p. 377).

\section{Referencias}

Bourdieu, P. (1972). Esquisse d'une theorie de la pratique. Genève, Paris: Droz.

Cárdenas, M. L. (2006). Bilingual Colombia. Are we ready for it? What is needed? Proceedings of the 19th Annual English Australia Education Conference, Perth, Australia. Recuperado el 13 de agosto de 2013 de: http:// www.academia.edu/1833795/Bilingual_Colombia_Are_we_ready_for_it_What_is_needed

Consejo de Bogotá (2006). Acuerdo 253 “Bogotá bilingüe". Bogotá: Autor. Recuperado el 13 de agosto de 2013 de: http://www.alcaldiabogota.gov.co/sisjur/normas/Norma1.jsp?i=22230

Correa, D. y Usma, J. (2013). From a burocratic to a critical-sociocultural model of policymaking in Colombia. HOW A Colombian journal of teachers of English, 20, 226-242. 
ISSN 0123-1294 | Educ.Educ. Vol. 17. No. 2 | Mayo-Agosto de 2014 | pp. 215-236.

Universidad de La Sabana | Facultad de Educación

Dávila, D. (2012). Capital lingüístico en inglés y desigualdad de oportunidades educativas: exploración en dos colegios oficiales de Bogotá. (Tesis inédita de maestría). Universidad Nacional de Colombia, Bogotá. Recuperado el 28 de agosto de 2013 de: http://www.bdigital.unal.edu.co/5679/

De Mejía, A. y Fonseca, L. (2008). Orientaciones para políticas bilingües y multilingües en lenguas extranjeras en Colombia. Bogotá: Centro de Investigación y Formación en Educación (CIFE). Recuperado el 28 de agosto de 2013 de: http://www.colombiaaprende.edu.co/html/home/1592/articles-251339_pdf.pdf

De Mejía, A. (1998). Educación bilingüe en Colombia en contextos lingüísticos mayoritarios: hacia una caracterización del campo. Revista del Lenguaje, 26, 8-23. Recuperado el 17 de julio de 2013 de: http://revistalenguaje.univalle.edu.co/index.php? seccion $=$ REVISTA\&revista $=26 \&$ articulo $=202$

De Mejía, A., Ordóñez, C. y Fonseca, L. (2006). Lineamientos para la educación bilingüe en Colombia: hacia una politica coherente. Informe de investigación. Bogotá: Centro de Investigación y Formación en Educación Universidad de los Andes.

Fandiño, Y., Bermúdez, J. y Lugo, V. (2012). Retos del Programa Nacional de Bilingüismo. Colombia Bilingüe. Educación y educadores, 15(3), 363-381. Recuperado el 17 de julio de 2013 de: http://educacionyeducadores.unisabana.edu.co/index.php/eye/article/view/2172/2912

Ferguson, C. A., Houghton, C. \& Wells, M. H. (1977). Bilingual education: An international perspective. En Spolsky, B. \& Cooper, R. (eds.). Frontiers of bilingual education (pp. 154-179). Rowley, MA: Newbury House.

Ferrández, A. (1991). Las teorías del currículum. En Medina, A. y Sevillano, Ma. L. (coords.). El currículum: fundamentación, diseño, desarrollo y evaluación (pp. 250-257). Madrid: UNED.

Galindo, A., y Moreno, L. (2008). Estructura, resultados y retos del programa de bilingüismo colombiano 20042019. Revista de investigaciones de la Universidad del Quindio, 18, 172-179.

García, J., y García, D. (2012). Políticas lingüísticas en Colombia: tensiones entre políticas para lenguas mayoritarias y lenguas minoritarias. Boletín de filología, 47(2), 47-70. Recuperado el 28 de junio de 2013 de: http:// www.scielo.cl/scielo.php?pid=S0718-93032012000200002\&script=sci_arttext

Habermas, J. (1990). Conocimiento e interés. Buenos Aires: Taurus.

Halbach, A. (2008). Una metodología para la enseñanza bilingüe en la etapa de primaria. Revista de Educación, 346, 455-466. Recuperado el 28 de junio de 2013 de: http://www.revistaeducacion.mec.es/re346/re346_17.pdf

Herazo, J., Jerez, S., \& Lorduy, D. (2012). Opportunity and incentive for becoming bilingual in Colombia: implications for Programa Nacional de Bilingüismo. ÍKALA Revista de lenguaje y cultura, 17(2), 199-213.

Jornada de la Mañana (tesis inédita de maestría). Pontificia Universidad Javeriana, Bogotá, Colombia. Recuperado el 16 de mayo de 2013 de: http://repository.javeriana.edu.co/bitstream/10554/1041/1/edu64.pdf 
Kroon, S. (2000). Language Policy Development in Multilingual Societies. En van der Meer, T. \& Den Elt, M. (eds.). Nationalities and Education: Perspectives in Policy making in Russia and the Netherlands (pp. 15-38). Utrecht: Sardes.

Ley 115. Ley General de Educación (1994). Diario Oficial, 41.214. Colombia. Recuperado el 16 de mayo de 2013 de: http://www.secretariasenado.gov.co/senado/basedoc/ley/1994/ley_0115_1994.html

Ley 1651. Ley de Bilingüismo (2013). Diario Oficial, 48.849. Colombia. Recuperado el 27 de junio de 2013 de: http:// www.secretariasenado.gov.co/senado/basedoc/ley/2013/ley_1651_2013.html

López, A., Peña, B. y Guzmán, M. (2011). Transición hacia el bilingüismo en colegios públicos colombianos: estudio de caso. En López, A., Peña, B. y Truscott, A. (eds.). Bilingüismo en el contexto colombiano: iniciativas y perspectivas en el siglo XXI (pp. 23-45). Bogotá: Ediciones Uniandes.

López, A., Peña, B., De Mejía, A., Fonseca, L. y Guzmán, M. (2009). Necesidades y políticas para la implementación de un programa bilingüe en colegios distritales. En Montoya, J. (ed.). Educación para el siglo XXI: aportes del Centro de Investigación y Formación en Educación 2001-2008 (pp. 409-466). Bogotá: Ediciones Uniandes. Recuperado el 11 de junio de 2013 de: http://pensandoeducacion.uniandes.edu.co/ponencias/Lopez_et_ al.-Bilinguismo_en_colegios_distritales.pdf

Malagón, L. (2008). El currículo: perspectivas para su interpretación. Investigación y Educación en Enfermería, 26(2), 136-142. Recuperado el 2 de julio de 2013 de: http://www.scielo.org.co/scielo.php?pid=So12O53072008000300013\&script=sci_arttext

Maturana, L. (2011). La enseñanza del inglés en tiempos del plan nacional de bilingüismo en algunas instituciones públicas: Factores lingüísticos y pedagógicos. Colombian applied linguistics journal, 13(2), 74-87. Recuperado el 10 de mayo de 2013 de: http://www.redaly c.org/articulo.oa?id=305726661006

Ministerio de Educación Nacional (2006). Estándares Básicos de Competencias en Lenguas Extranjeras: Inglés. Bogotá: MEN.

Ministerio de Educación Nacional (2007). Plan Nacional Decenal de Educación 2006-2016. Bogotá: MEN.

Ministerio de Educación Nacional (s.f). Programa Nacional de Bilingüismo. Bogotá: MEN. Recuperado el 8 de mayo de 2013 de: http://www.colombiaaprende.edu.co/html/productos/1685/article-158720.html\#h2_1

Miranda, I. R. (2012). Insights on bilingualism and bilingual education: A sociolinguistic perspective. ÍKALA, revista de lenguaje y cultura, 17(3) 263-272. Recuperado el 9 de abril de 2013 de: http://www.redaly c.org/ articulo.oa?id=255025411004

Moreno, A. y Ramírez, J. (2003). Pierre Bourdieu: Introducción elemental. Bogotá: Panamericana.

Moreno, M. C. (2009). Análisis de la implementación de las políticas públicas de bilingüismo en Bogotá (tesis inédita de maestría). Universidad de La Salle, Bogotá, Colombia. 
ISSN 0123-1294 | Educ.Educ. Vol. 17. No. 2 | Mayo-Agosto de 2014 | pp. 215-236.

Universidad de La Sabana | Facultad de Educación

Nunan, D. (2003). Practical English language teaching. USA: McGraw-Hill Company.

Pal, S. (2010). Análisis de la Implementación del "Programa de Transición Hacia el Bilingüismo 2004-2019 en Bogotá y Cundinamarca” en el Colegio Distrital Cundinamarca IED.

Peña, B., Flaborea, R. y Guzmán, M. (2011). Propuesta para el fortalecimiento del área de inglés en un colegio no bilingüe de Bogotá. Apuntes desde la experiencia de un estudio de caso. En López, A., Peña, B.y De Mejía, A. (eds.). Bilingüismo en el contexto colombiano: iniciativas y perspectivas en el siglo XXI (pp. 115-165). Bogotá: Ediciones Uniandes.

Rodríguez, A. (2011). Evaluación de la politica de bilingüismo en Bogotá: Proyecto "Bogotá bilingüe" (tesis inédita de maestría). Pontificia Universidad Javeriana, Bogotá, Colombia. Recuperado el 12 de mayo de 2013 de: http://repository.javeriana.edu.co/bitstream/10554/1569/1/RodriguezGarciaAlixRocio2011.pdf

Secretaría de Educación de Bogotá (2004). Plan Sectorial de Educación 2004-2008 "Bogotá: una gran escuela". Bogotá: Alcaldía Mayor de Bogotá.

Secretaría de Educación de Bogotá (2008). Plan Sectorial de Educación 2008-2012 "Educación de calidad para una Bogotá Positiva". Bogotá: Alcaldía Mayor de Bogotá.

Secretaría de Educación de Bogotá (2012). Plan Sectorial de Educación 2012-2016 “Bogotá Humana”. Bogotá: Alcaldía Mayor de Bogotá.

Shohamy, E. (2009). Language Teachers as Partners in Crafting Educational Language Policies? ÍKALA, Revista de lenguaje y cultura, 14(22), 45-67. Recuperado el 19 de abril de 2013 de: http://www.redaly c.org/articulo. oa? id $=255020476002$

Siguan, M. (1986). Educación y Bilingüismo. Madrid: Santillana.

Spolsky, B. (2004). Language Policy. United Kingdom: Cambridge University Press.

Stenhouse, L. (1985). Investigación y desarrollo del currículum. Madrid: Morata.

Torres-Martínez, S. (2009). Las vicisitudes de la enseñanza de lenguas en Colombia. Diálogos latinoamericanos, 15, 56-75. Recuperado 10 de abril de 2013 de: http://www.redaly c.org/articulo.oa?id=16220868004

Usma, W. (2009). Education and language policy in Colombia: exploring processes of inclusion, exclusion, and stratification in times of global reform. PROFILE Issues in teachers' professional development, 11, 123-141.

Vargas, A., Tejada, H. y Colmenares, S. (2008). Estándares básicos de competencias en lenguas extranjeras (inglés): una lectura crítica. Lenguaje: Revista de la Escuela de Ciencias del Lenguaje de la Universidad del Valle, 36(1), 241-275.

Vieira, I. \& Moreira, M. A. (2008). Reflective teacher education towards learner autonomy: building a culture of possibility. En Raya, M. \& Lamb, T. (eds.). Pedagogy for Autonomy in Language Education: Theory, practice and teacher education (pp. 266-282). Dublin: Authentik. 